УДК 54.057+544.526.1

DOI: $10.33184 /$ bulletin-bsu-2020.3.9

\title{
ФОТОХИМИЧЕСКАЯ АКТИВАЦИЯ ПРОЦЕССА ВОССТАНОВЛЕНИЯ
} $\mathrm{Sm}^{3+}$ ДО $\mathrm{Sm}^{2+}$ В СИСТЕМЕ $\mathrm{SmCl}_{3} \cdot 6 \mathrm{H}_{2} \mathrm{O}-{ }^{i} \mathrm{Bu}_{2} \mathrm{AlH}-$ ТГФ

(С Д. И. Галимов ${ }^{1 *}$, С. М. Якупова ${ }^{1}$, Р. Г. Булгаков ${ }^{2}$

${ }^{1}$ Институт нефтехимии и катализа УФИЦ РАН

Россия, Республика Башкортостан, 450075 г. Уфа, пр. Октября, 141.

${ }^{2}$ Институт физики молекул и кристаллов УФИЦ РАН

Россия, Республика Башкортостан, 450054 г. Уфа, пр. Октября, 71.

Tел./факс: +7 (347) 2842750.
*Email: galimovdi@ mail.ru

Впервые изучена способность трехвалентных ионов лантанидов $\mathrm{Ln}^{3+}(\mathrm{Ln}=\mathrm{Sm}, \mathrm{Tm}, \mathrm{Dy}, \mathrm{Nd}) \kappa$ восстановлению до двухвалентного состояния при взаимодействии с алюминийорганическими соединенияли в среде апротонного растворителя тетрагидрофурана без и при дополнительном воздействии микроволнового, ультрафиолетового и ультразвукового облучения. Обнаружено, что процесс восстановления самария $\mathrm{Sm}^{3+}$ до $\mathrm{Sm}^{2+}$ под действием диизобутилалюминийгидрида ${ }^{i} B u_{2} A l H$, характеризующийся относительной длительностью процесса - от 2 часов до суток, значительно ускоряется при УФ-облучении и осуществляется за рекордные 15 минут. Прочесс восстановления контролировался по спектрам поглощения и фотолюминесиенции $\mathrm{Sm}^{2+}$ в реакиионном растворе. Установлено, что восстановление других ионов лантанидов $\mathrm{Ln}^{3+}(\mathrm{Ln}=\mathrm{Tm}, \mathrm{Dy}, \mathrm{Nd})$ при действии ${ }^{i} B u_{2} \mathrm{AlH}$ без и в сочетании с облучением не происходит, что связано с большими отрицательными восстановительными потенциалами $E^{0}\left(\operatorname{Ln}^{3+} / \mathrm{Ln}^{2+}\right) \leq-2.27$ В для указанных ионов лантанидов.

Ключевые слова:ион $\mathrm{Sm}^{2+}$, фотохимический синтез, восстановление $\mathrm{Sm}^{3+}$, фотолюминесиенция, УФ-облучение.

\section{Введение}

Соединения двухвалентного самария находят широкое применение в органическом синтезе в качестве катализаторов и одноэлектронных восстановителей [1-4], а также в технике в качестве люминесцентных материалов с заданными спектральноэнергетическими свойствами [5-6]. Особенно большое практическое применение как селективный мягкий восстановитель получил раствор дийодида самария $\mathrm{SmI}_{2}$ в тетрагидрофуране (ТГФ), известный как «реагент Кагана» [1]. Что касается люминесцентных свойств двухвалентного самария, то наиболее изученной является фотолюминесценция (ФЛ) иона $\mathrm{Sm}^{2+}$, допированного в различные твердые неорганические матрицы [7-8]. Гораздо менее исследованы спектрально-люминесцентные характеристики соединений $\mathrm{Sm}^{2+}$ в растворе [9-11], что обусловлено низким квантовым выходом люминесценции и нестабильностью иона $\mathrm{Sm}^{2+}$, вызванной действием окислителей - воды и кислорода воздуха.

В настоящее время соединения двухвалентного самария получают путем восстановления трехвалентного самария в реакции $\mathrm{Sm}_{2} \mathrm{O}_{3}$ с хлорорганическими соединениями в атмосфере водорода при 600-1500 ${ }^{\circ} \mathrm{C}$ [12-13], при взаимодействии металлического самария $\mathrm{Sm}^{0}$ с дийодэтаном или дийодметаном в среде ТГФ при комнатной температуре в течении 24 ч [1] или с молекулярным йодом при 600-800 ${ }^{\circ} \mathrm{C}$ [14]. Таким образом, известные способы получения соединений $\mathrm{Sm}^{2+}$ характеризуются наличием ряда недостатков: взрывоопасностью процессов [14], применением высоких температур [12-14] и агрессивной среды [12-13], длительностью процесса восстановления [1]. Простым и эффективным способом получения $\mathrm{SmCl}_{2}$ является восстановление $\mathrm{Sm}^{3+}$ до $\mathrm{Sm}^{2+}$ при взаимодействии коммерчески доступного кристаллогидрата трихлорида самария $\mathrm{SmCl}_{3} \cdot 6 \mathrm{H}_{2} \mathrm{O}$ с алюминийорганическими соединениями (АОС) [15]. Восстановление осуществляется в достаточно мягких условиях (комнатная температура, атмосферное давление), но отличается значительной длительностью процесса (от 2 до 24 ч). С целью ускорения восстановления $\mathrm{Sm}^{3+} \rightarrow \mathrm{Sm}^{2+}$ в настоящей работе изучено влияние ультрафиолетового (УФ), ультразвукового (УЗ) и микроволнового (МВ) излучения на процесс восстановления самария в реакции $\mathrm{SmCl}_{3} \cdot 6 \mathrm{H}_{2} \mathrm{O}$ с рядом AOC: ${ }^{i} \mathrm{Bu}_{2} \mathrm{AlH},{ }^{i} \mathrm{Bu}_{3} \mathrm{Al}, \mathrm{Et}_{3} \mathrm{Al}$ в среде ТГФ. Стимулом для применения этих излучений для восстановления самария явились результаты работ по ускорению процесса восстановления облучением растворов соединений ионов разных металлов $\mathrm{Me}^{n+} \rightarrow \mathrm{Me}^{(n-1)+}$, где $\mathrm{Me}=\mathrm{Fe}, \mathrm{Eu}, \mathrm{Pb}, \mathrm{Au}$ [16-19].

\section{Экспериментальная часть}

В работе использованы гексагидрат трихлорида самария $\mathrm{SmCl}_{3} \cdot 6 \mathrm{H}_{2} \mathrm{O}$ марки «ХЧ», который для удаления адсорбированной воды предварительно держали в вакууме ( 1 мм рт. ст.) в течение 1 ч при комнатной температуре. Толуольные растворы AOC: ${ }^{i} \mathrm{Bu}_{2} \mathrm{AlH}(73 \%),{ }^{i} \mathrm{Bu}_{3} \mathrm{Al}(93 \%)$ и $\mathrm{Et}_{3} \mathrm{Al}(93 \%)$ применяли без дополнительной очистки. В качестве растворителя выбран ТГФ марки «ХЧ», который под- 
вергали ступенчатой очистке путем выдержки и кипячения над $\mathrm{KOH} \rightarrow \mathrm{CaH}_{2} \rightarrow \mathrm{Na}^{0} \rightarrow \mathrm{LiAlH}_{4}$ согласно [20]. Аргон из баллона марки «А» $(99.99 \%)$ доочищали пропусканием через прибор «Поглотитель газов».

Стандартная процедура проведения реакции заключалась в следующем. В стеклянный пирексевый сосуд Шленка объемом 10 мл, установленный на магнитную мешалку, помещали кристаллогидрат $\mathrm{SmCl}_{3} \cdot 6 \mathrm{H}_{2} \mathrm{O}$ (0.02 ммоль, 7.30 мг) и 2 мл свежеперегнанного ТГФ. Далее при перемешивании в атмосфере аргона добавляли 0.8 ммоль АОС. При этом наблюдается сильное газовыделение, быстрое исчезновение твердой фазы и образование бесцветного гомогенного раствора, который подвергали УЗ, УФ или МВ облучению. Завершение процесса восстановления контролировали измерением спектров ФЛ и поглощения. УФ и УЗ облучение реакционных растворов проводили облучателем «Квант» (светофильтр УФС-2, дуговая ртутно-шаровая лампа ДРШ-500 Вт) и ультразвуковым генератором ИЛ-10-0.63 (600 Вт) с погружным титановым волноводом, соответственно. Постоянную температуру (20-25 $\left.{ }^{\circ} \mathrm{C}\right)$ реакционного раствора поддерживали охлаждением сосуда Шленка медным змеевиком с проточной водой. МВ облучение реакционных растворов проводили в запаянных ампулах в бытовой микроволновой печи мощностью 700 Вт.

Для выделения комплекса $\mathrm{Sm}^{2+}$ из реакционного раствора, полученного (в результате и без облучения) после взаимодействия в системе $\mathrm{SmCl}_{3} \cdot 6 \mathrm{H}_{2} \mathrm{O}{ }^{i} \mathrm{Bu}_{2} \mathrm{AlH}-$ ТГФ, сначала удаляли растворитель (ТГФ) путем вакуумной отгонки (5-6 мм рт. ст.) при 20-25 ${ }^{\circ} \mathrm{C}$. Затем к жидкому остатку добавляли $\sim 3$ мл гексана; образующийся при этом осадок светло-желтого цвета промывали толуолом или гексаном $(5 \times 2$ мл) до полного удаления избыточного АОС и продуктов его превращения. Отсутствие ионов $\mathrm{Al}^{3+}$ в маточном растворе контролировали методами прямого и обратного комплексонометрического титрования [20]. Полученный после отмывки твердый остаток сушили в вакууме (не более $40{ }^{\circ} \mathrm{C}, 1$ мм рт.ст.). В результате получен порошок светло-желтого цвета. Состав этого порошка установлен с применением комплексонометрического и элементного анализов, a также ИК-спектроскопии. По результатам элементного анализа состав комплекса соответствует брутто-формуле $\mathrm{C}_{8} \mathrm{H}_{16} \mathrm{O}_{2} \mathrm{SmCl}_{2}\left(\mathrm{SmCl}_{2} \cdot(\mathrm{THF})_{2}\right)$. Наличие в координационной сфере самария молекул ТГФ подтверждают ИК-спектры, в которых полосы ТГФ, координированного с $\mathrm{Sm}^{2+}$, сдвинуты на $\sim 48 \mathrm{~cm}^{-1}$ по сравнению со свободным ТГФ.

$\mathbf{S m C l}_{2} \cdot(\mathbf{T H F})_{2}$. Твердое вещество светло-желтого цвета. Рассчитано для $\mathrm{C}_{8} \mathrm{H}_{16} \mathrm{O}_{2} \mathrm{SmCl}_{2}(\%)$ : $\mathrm{Sm}-$ 41.98; Cl - 19.45; C - 26.3; Н - 4.98; O - 8.77. Найдено (\%): $\mathrm{Sm}-40.32 ; \mathrm{Cl}-19.06 ; \mathrm{C}-26.46 ; \mathrm{H}-4.66$; $\mathrm{O}-9.50$. УФ спектр $(0.65 \mathrm{M} \mathrm{HCl}) \lambda_{\max } /$ нм: 350,382 , 417. ИК спектр (KBr), v/см ${ }^{-1}: 872$ сл. (С-О, ТНF); 1030 сл. (C-O, THF); 684 cp. (Sm-Cl). Выход комплекса составил $40 \%$ от теоретического.
Спектры возбуждения и ФЛ регистрировали в кварцевых герметичных кюветах $(l=1$ см) на спектрофлуориметре FluoroLog-3 Horiba Jobin Yvon. Для регистрации ФЛ твердых образцов $\mathrm{SmCl}_{3} \cdot 6 \mathrm{H}_{2} \mathrm{O}$ и $\mathrm{SmCl}_{2} \cdot(\mathrm{THF})_{2}$ применяли держатель твердых образцов с тонким покровным кварцевым стеклом, расположенным под углом 60 градусов к возбуждающему свету, а растворов - в кварцевой кювете $(l=1 \mathrm{~cm})$ с тефлоновой крышкой (облучение под углом 90 градусов). УФ спектры поглощения растворов регистрировали в герметичных кварцевых кюветах $(l=1 \mathrm{~cm})$ на спектрофотометре Shimadzu UV-1800, а ИК спектры твердых образцов в таблетках $\mathrm{KBr}$ измеряли на приборе Bruker Vertex $70 \mathrm{~V}$. Элементный состав (C, H, О) комплекса $\mathrm{SmCl}_{2} \cdot(\mathrm{THF})_{2}$ определяли на элементном анализаторе фирмы «CarloErba».

\section{Результаты и их обсуждение}

Исходная соль $\mathrm{SmCl}_{3} \cdot 6 \mathrm{H}_{2} \mathrm{O}$ не растворяется в ТГФ и образует взвесь. При добавлении к этой взвеси аликвоты АОС наблюдается исчезновение твердой фазы с образованием прозрачного гомогенного раствора. Одновременно в результате реакции АОС с кристаллизационной водой соли $\mathrm{SmCl}_{3} \cdot 6 \mathrm{H}_{2} \mathrm{O}$ бурно выделяются газообразные продукты (согласно данным ГЖХ и ГХ): алканы ${ }^{i} \mathrm{BuH}$ и EtH (в случае ${ }^{i} \mathrm{Bu}_{3} \mathrm{Al}$ и $\mathrm{Et}_{3} \mathrm{Al}$ ) или $\mathrm{H}_{2}$ и ${ }^{i} \mathrm{BuH}$ (для ${ }^{i} \mathrm{Bu}_{2} \mathrm{AlH}$ ). Реакционный раствор остается прозрачным в течение 1.5 ч от начала реакции. Затем он постепенно приобретает интенсивную сине-зеленую окраску, характерную для иона двухвалентного самария $[9 ; 21]$. Кроме изменения цвета, факт восстановления $\mathrm{Sm}^{3+} \rightarrow \mathrm{Sm}^{2+}$ подтверждает существенное отличие спектров поглощения и ФЛ исходного кристаллогидрата $\mathrm{SmCl}_{3} \cdot 6 \mathrm{H}_{2} \mathrm{O}$ и реакционного раствора. Так, спектр ФЛ кристаллического образца $\mathrm{SmCl}_{3} \cdot 6 \mathrm{H}_{2} \mathrm{O}$ состоит из узких полос (рис. 1), характерных [22] для излучательных $f f$-переходов трехвалентных ионов лантанидов. После взаимодействия $\mathrm{SmCl}_{3} \cdot 6 \mathrm{H}_{2} \mathrm{O}$ с AOC эти максимумы в спектре ФЛ исчезают, и появляется одна широкая полоса $f d$-люминесценции иона $\mathrm{Sm}^{2+}$ (рис. 1), положение которой хорошо согласуется с литературными данными [9; 23]. Спектр поглощения полученного гомогенного раствора также содержит характерные полосы иона $\mathrm{Sm}^{2+}$ при 350 , 382 и 417 нм [9; 23].

Изучение кинетики восстановления $\mathrm{Sm}^{3+} \rightarrow \mathrm{Sm}^{2+}$ по возрастанию оптической плотности полосы поглощения при 417 нм без применения какого-либо облучения показало, что время полного восстановления трехвалентного самария до двухвалентного состояния в реакции $\mathrm{SmCl}_{3} \cdot 6 \mathrm{H}_{2} \mathrm{O}$ с ${ }^{i} \mathrm{Bu}_{2} \mathrm{AlH}$ составляет в среднем 2 ч (рuc. 2).

Кинетика восстановления самария кардинально меняется при УФ облучении гомогенного реакционного раствора, образующегося после взаимодействия в системе $\mathrm{SmCl}_{3} \cdot 6 \mathrm{H}_{2} \mathrm{O}-{ }^{i} \mathrm{Bu}_{2} \mathrm{AlH}-\mathrm{TГ} \Phi$, т.к. полное восстановление $\mathrm{Sm}^{3+}$ до $\mathrm{Sm}^{2+}$ осуществляется за рекордные 15 мин (рис. 2). 


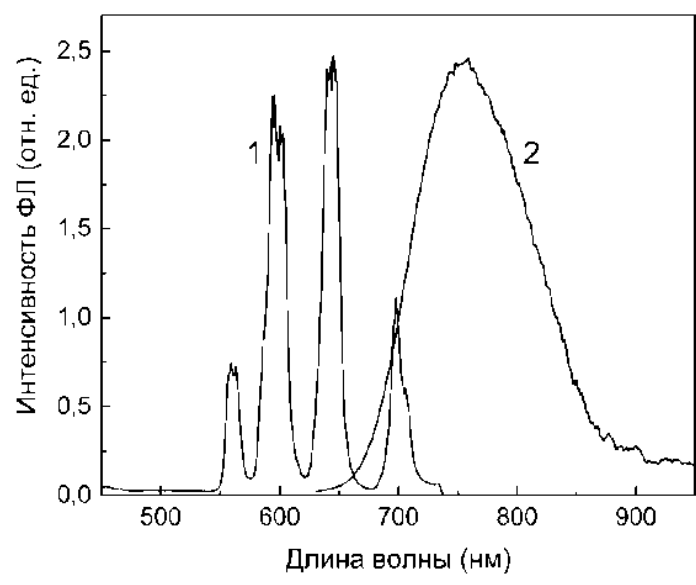

Рис 1. Спектры ФЛ кристаллического образца $\mathrm{SmCl}_{3} \cdot 6 \mathrm{H}_{2} \mathrm{O}$ (1) и реакционного раствора (2), полученного при взаимодействии $\mathrm{SmCl}_{3} \cdot 6 \mathrm{H}_{2} \mathrm{O}$ (0.02 ммоль) $\mathrm{c}^{i} \mathrm{Bu}_{2} \mathrm{AlH}$ (0.8 ммоль) в ТГФ (2 мл); $\lambda_{\text {возо }}=408$ (1), 480 (2) нМ; $T=298 \mathrm{~K}$.

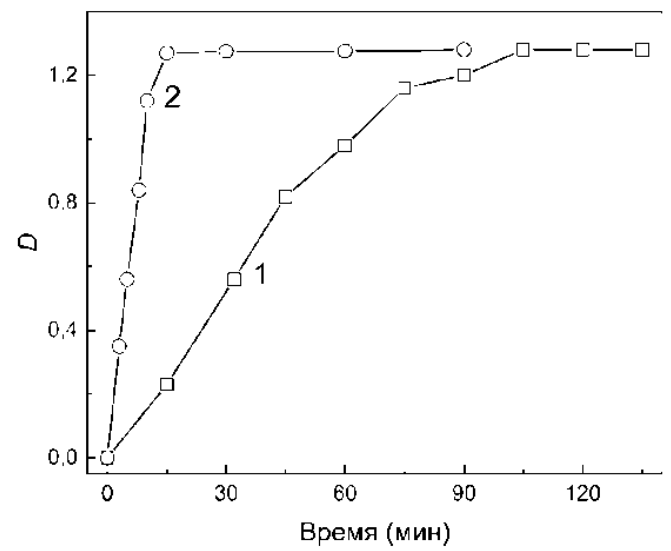

Рис. 2. Кинетика восстановления $\mathrm{Sm}^{3+} \rightarrow \mathrm{Sm}^{2+}$ при взаимодействии $\mathrm{SmCl}_{3} \cdot 6 \mathrm{H}_{2} \mathrm{O}$ (0.02 ммоль) $\mathrm{c}^{i} \mathrm{Bu}_{2} \mathrm{AlH}$ (0.8 ммоль) в ТГФ (2 мл) без (1) и при облучении УФ-светом (2); $l=1 \mathrm{~cm} ; T=298 \mathrm{~K}$.

Сравнение этих результатов с полученными ранее в работах [1,11-15] по синтезу $\mathrm{SmX}_{2} \cdot(\mathrm{THF})_{2}$ (где $\mathrm{X}=\mathrm{I}, \mathrm{Cl}$ ) показывает, что при использовании УФ-облучения и гидрида ${ }^{i} \mathrm{Bu}_{2} \mathrm{AlH}$ в качестве восстановителя действительно достигается рекордно короткое время синтеза.

В отличии от УФ облучения, УЗ и МВ облучение такого же реакционного раствора в аналогичных условиях никакого влияния на процесс восстановления самария не оказало.

Как оказалось, на процесс фотохимического восстановления самария существенно влияет природа АОС. Действительно, в отличие от ${ }^{i} \mathrm{Bu}_{2} \mathrm{AlH}$, при применении незамещенных алюминийалкилов ${ }^{i} \mathrm{Bu}_{3} \mathrm{Al}$ и $\mathrm{Et}_{3} \mathrm{Al}$ процесс восстановления требует гораздо большего времени (около 24 ч). Еще одной важной отличительной особенностью использования ${ }^{\mathrm{i}} \mathrm{Bu}_{3} \mathrm{Al}$ и $\mathrm{Et}_{3} \mathrm{Al}$ в качестве восстановителей, является отсутствие ускорения процесса восстановле- ния при УФ облучении реакционных растворов, полученных после взаимодействия в системах $\mathrm{SmCl}_{3} \cdot 6 \mathrm{H}_{2} \mathrm{O}-{ }^{i} \mathrm{Bu}_{3} \mathrm{Al}-$ ТГФ и $\mathrm{SmCl}_{3} \cdot 6 \mathrm{H}_{2} \mathrm{O}-\mathrm{Et}_{3} \mathrm{Al}-$ ТГФ. Сравнение состава этих систем и системы $\mathrm{SmCl}_{3} \cdot 6 \mathrm{H}_{2} \mathrm{O}-{ }^{i} \mathrm{Bu}_{2} \mathrm{AlH}-\mathrm{TГ} Ф$ позволяет сделать однозначный вывод о том, что необходимым условием для достижения эффекта фотохимического ускорения восстановления самария при УФ облучении является наличие в АОС гидридной связи Al-H.

На основании результатов, полученных в данной работе, а также в наших, более ранних исследованиях механизма взаимодействия кристаллогидратов лантанидов $\mathrm{LnCl}_{3} \cdot 6 \mathrm{H}_{2} \mathrm{O}(\mathrm{Ln}=\mathrm{Eu}, \mathrm{Yb}, \mathrm{Sm})$ с АОС в ТГФ без УФ облучения $[11 ; 15 ; 20]$ механизм химического восстановления $\mathrm{Sm}^{3+} \rightarrow \mathrm{Sm}^{2+}$ и его фотохимической активации при взаимодействии кристаллогидрата $\mathrm{SmCl}_{3} \cdot 6 \mathrm{H}_{2} \mathrm{O}$ с ${ }^{i} \mathrm{Bu}_{2} \mathrm{AlH}$ в ТГФ можно представить в виде следующей вероятной схемы.

Взаимодействие в системе $\mathrm{SmCl}_{3} \cdot 6 \mathrm{H}_{2} \mathrm{O}-$ ${ }^{i} \mathrm{Bu}_{2} \mathrm{AlH}-\mathrm{TГФ} \mathrm{начинается} \mathrm{с} \mathrm{атаки}{ }^{i} \mathrm{Bu}_{2} \mathrm{AlH}$ на молекулы кристаллизационной воды $\mathrm{SmCl}_{3} \cdot 6 \mathrm{H}_{2} \mathrm{O}$ (cxeма, реакция 1) с образованием газообразных продуктов ( $\left.{ }^{i} \mathrm{BuH}, \mathrm{H}_{2}\right)$, а также растворимых в ТГФ алюмоксана $\left({ }^{i} \mathrm{Bu}_{2} \mathrm{Al}\right)_{2} \mathrm{O}$ и комплекса трехвалентного самария $\mathrm{SmCl}_{3} \cdot(\mathrm{THF})_{x}$. Возможность образования такого комплекса трехвалентного лантанида доказана на примере взаимодействия в системе $\mathrm{TbCl}_{3} \cdot 6 \mathrm{H}_{2} \mathrm{O}-{ }^{i} \mathrm{Bu}_{2} \mathrm{AlH}-\mathrm{TГ} Ф$, т.к. ион $\mathrm{Tb}^{3+}$ не восстанавливается ${ }^{i} \mathrm{Bu}_{2} \mathrm{AlH}$ из-за крайне отрицательного восстановительного потенциала пары $E^{0}\left(\mathrm{~Tb}^{3+} / \mathrm{Tb}^{2+}\right)=-3.7$ В [24]. В тоже время ион $\mathrm{Tb}^{3+}$ после взаимодействия в этой системе также образует хорошо растворимый в ТГФ комплекс $\mathrm{TbCl}_{3} \cdot(\mathrm{THF})_{2}$, выделенный и охарактеризованный нами методами ФЛ, комплексонометрии, элементного анализа, УФ-, ИК-спектроскопии [11]. На образование комплекса $\mathrm{SmCl}_{3}$. (THF) $)_{x}$ также указывает тот факт, что без облучения $\mathrm{Sm}^{3+}$ восстанавливается до $\mathrm{Sm}^{2+}$ более, чем за 2 ч, а исчезновение («растворение») твердой соли $\mathrm{SmCl}_{3} \cdot 6 \mathrm{H}_{2} \mathrm{O}$ в ТГФ при добавлении ${ }^{i} \mathrm{Bu}_{2} \mathrm{AlH}$ (или других $\mathrm{AOC}$ ) с образованием прозрачного гомогенного раствора происходит практически мгновенно. Кратко резюмируя, гомогенный раствор трехвалентного лантанида образуется сразу после добавления АОС, а восстановление продолжается значительно дольше.

На следующей стадии (схема, реакция 2) под действием избытка ${ }^{i} \mathrm{Bu}_{2} \mathrm{AlH}$ по механизму переалкилирования ион $\mathrm{Sm}^{3+}$ восстанавливается до $\mathrm{Sm}^{2+}$ с образованием комплекса $\mathrm{SmCl}_{2} \cdot(\mathrm{THF})_{x}$ (где $x \leq 5$ [25]) и диизобутилалюминий хлорида ${ }^{i} \mathrm{Bu}_{2} \mathrm{AlCl}$, образование которого доказано нами с применением ЯМР ${ }^{1} \mathrm{H}\left(\mathrm{CDCl}_{3}, \delta\right.$, м.д.): 1.34 (m, 4H, $\left.\mathrm{CH}_{2}\right) ; 3.19$. 


$$
\begin{aligned}
& \mathrm{SmCl}_{3} \cdot 6 \mathrm{H}_{2} \mathrm{O}_{(\text {тв })}+{ }^{\mathrm{i}} \mathrm{Bu}_{2} \mathrm{AlH} \stackrel{\text { ТГФ }}{\longrightarrow} \mathrm{SmCl}_{3} \cdot(\mathrm{THF})_{\mathrm{x}}+\left({ }^{\mathrm{i}} \mathrm{Bu}_{2} \mathrm{Al}\right)_{2} \mathrm{O}+{ }^{\mathrm{i}} \mathrm{BuH}+\mathrm{H}_{2} \\
& \mathrm{SmCl}_{3} \cdot(\mathrm{THF})_{\mathrm{x}}+{ }^{\mathrm{i}} \mathrm{Bu}_{2} \mathrm{AlH} \longrightarrow\left[\mathrm{HSmCl}_{2} \cdot(\mathrm{THF})_{\mathrm{x}}-{ }^{\mathrm{i}} \mathrm{Bu}_{2} \mathrm{AlCl}\right] \stackrel{\text { медленно }}{-\mathrm{H}} \mathrm{SmCl}_{2} \cdot(\mathrm{THF})_{\mathrm{x}}+{ }^{\mathrm{i}} \mathrm{Bu}_{2} \mathrm{AlCl} \\
& \left.\mathrm{SmCl}_{2} \cdot(\mathrm{THF})_{\mathrm{x}}+{ }^{\mathrm{i}} \mathrm{Bu}_{2} \mathrm{AlH} \text { (избыток }\right) \longrightarrow \mathrm{SmCl}_{2} \cdot(\mathrm{THF})_{\mathrm{x}}-{ }^{\mathrm{i}} \mathrm{Bu}_{2} \mathrm{AlH} \\
& \mathrm{SmCl}_{2} \cdot(\mathrm{THF})_{\mathrm{x}}{ }^{\mathrm{i}} \mathrm{Bu}_{2} \mathrm{AlH} \stackrel{\text { гексан, толуол }}{\longrightarrow} \mathrm{SmCl}_{2} \cdot(\mathrm{THF})_{2} \\
& { }^{\mathrm{i}} \mathrm{Bu}_{2} \mathrm{AlH} \longrightarrow \mathrm{h} v \\
& \mathrm{SmCl}_{3} \cdot(\mathrm{THF})_{\mathrm{x}}{ }^{\mathrm{i}}{ }^{\mathrm{B}} \mathrm{Bu}_{2} \mathrm{AlH}+\mathrm{Bu}\left(\mathrm{H}_{2}\right) \stackrel{\text { быстро }}{\longrightarrow} \mathrm{SmCl}_{2} \cdot(\mathrm{THF})_{\mathrm{x}}-{ }^{\mathrm{i}} \mathrm{Bu}_{2} \mathrm{AlH}+\mathrm{HCl}\left(\mathrm{H}_{2}\right)
\end{aligned}
$$

$\left(\mathrm{m}, 4 \mathrm{P}, \mathrm{O}-\mathrm{CH}_{2}\right)$ и ${ }^{13} \mathrm{C}\left(\mathrm{CDCl}_{3}, \delta\right.$, м.д.): $25.00\left(\beta-\mathrm{CH}_{2}\right.$, THF); 66.99 ( $\left.\alpha-\mathrm{CH}_{2}, \mathrm{THF}\right)$.

Мы полагаем, что комплекс $\mathrm{SmCl}_{2} \cdot(\mathrm{THF})_{x}$ в реакционном растворе находится в виде более сложных комплексов $\mathrm{SmCl}_{2} \cdot(\mathrm{THF})_{x}-{ }^{i} \mathrm{Bu}_{2} \mathrm{AlCl}$ и $\mathrm{SmCl}_{2} \cdot(\mathrm{THF})_{x}{ }^{i} \mathrm{Bu}_{2} \mathrm{AlH}$. Последний образуется при взаимодействии комплекса $\mathrm{SmCl}_{2} \cdot(\mathrm{THF})_{x}$ с избыточным ${ }^{i} \mathrm{Bu}_{2} \mathrm{AlH}$ (схема, реакция 3 ). Скорее всего, эти комплексы имеют мостиковую четырехцентровую структуру, содержащую связи $\mathrm{Sm}-\mathrm{Cl}-\mathrm{Al}$, a также $\mathrm{Sm}-\mathrm{H}\left({ }^{i} \mathrm{Bu}\right)-\mathrm{Al}$, характерную для лантанидных катализаторов Циглера-Натты. Подобные структуры ранее были идентифицированы в качестве промежуточных продуктов взаимодействия соединений лантанидов с алюминийалкилами и их гидридами [26-28]. Также возможность образования подобных мостиковых четырехцентровых структур, включающих двухвалентный лантанид и алюминий, описана в работе Эванса [25].

Выделение твердого комплекса $\mathrm{SmCl}_{2} \cdot(\mathrm{THF})_{2}$ из раствора объемного комплекса $\mathrm{SmCl}_{2} \cdot(\mathrm{THF})_{x}-$ ${ }^{i} \mathrm{Bu}_{2} \mathrm{AlH}$ в ТГФ отражено в виде стадии 4 (схема). Отметим, что потеря части координированных молекул ТГФ, как при выделении, так и хранении подобных твердых комплексов, характерна для таких сольватированных комплексов, изначально содержащих три-пять молекул ТГФ [25; 29-31].

Мы полагаем, что при УФ облучении раствоpa, полученного после взаимодействия в системе $\mathrm{SmCl}_{3} \cdot 6 \mathrm{H}_{2} \mathrm{O}-{ }^{i} \mathrm{Bu}_{2} \mathrm{AlH}-\mathrm{TГФ,} \mathrm{происходит} \mathrm{фотолиз}$ связи $\mathrm{Al}-\mathrm{H}$ гидрида ${ }^{i} \mathrm{Bu}_{2} \mathrm{AlH}$ с образованием радикалов ${ }^{i} \mathrm{Bu}_{2} \mathrm{Al}^{*}$ и $\mathrm{H}^{*}$ (схема, реакция 5). $\mathrm{K}$ сожалению, в литературе мы не нашли работ по фотолизу ${ }^{i} \mathrm{Bu}_{2} \mathrm{AlH}$, имеются лишь сведения по фотолизу алюминийалкилов, например, $\mathrm{Et}_{3} \mathrm{Al}$ [32]. Здесь было обнаружено, что кроме алкильных радикалов $\mathrm{Et} t^{\bullet}$ образуется и молекулярный водород. Учитывая, что в нашем случае УФ облучение при восстановлении $\mathrm{Sm}^{3+}$ алюминийалкилами не дает эффекта ускорения, мы полагаем, что молекулы водорода при облучении алкилсодержащих систем не образуются. Образование атомарного водорода в работе [32] связано с применением для облучения жесткого вакуумного ультрафиолетового света $\left(\lambda_{\max }=193 \mathrm{HM}\right)$. В тоже время возможность образования атомов и молекул водорода в реакции (5) подтверждает факт их образования при фотолизе твердого алюмогидрида лития $\mathrm{LiAlH}_{4}$ обычной ртутной лампой $\left(\lambda_{\max }=365\right.$ нм) [33].

Радикалы и молекулы водорода далее восстанавливают трехвалентный самарий до двухвалентного состояния, атакуя объемный комплекс $\mathrm{SmCl}_{3} \cdot(\mathrm{THF})_{x}{ }^{-} \mathrm{Bu}_{2} \mathrm{AlH}$ (схема, реакция 6). Конечно, одновременно с быстрым фотохимическим восстановлением самария по реакции (6) идет и гораздо более медленное, чисто химическое восстановление по (2). Оценка соотношения скоростей этих процессов по соотношению скоростей реакции при достижении максимальной концентрации $\mathrm{Sm}^{2+}$ дает величину, равную 76.5 .

Полученные положительные результаты по УФ активации процесса восстановления самария в системе $\mathrm{SmCl}_{3} \cdot 6 \mathrm{H}_{2} \mathrm{O}-{ }^{i} \mathrm{Bu}_{2} \mathrm{AlH}-\mathrm{TГ}$, стимулировали нас к изучению возможности восстановления других ионов лантанидов $\mathrm{Tm}^{3+}, \mathrm{Dy}^{3+}$ и $\mathrm{Nd}^{3+}$, обладающих более отрицательными восстановительными потенциалами, чем $\mathrm{Sm}^{3+}$ (-1.55 В отн. НВЭ) [24]. Согласно [24] восстановительные потенциалы (для пар $\mathrm{Tm}^{3+} / \mathrm{Tm}^{2+}, \mathrm{Dy}^{3+} / \mathrm{Dy}^{2+}, \mathrm{Nd}^{3+} / \mathrm{Nd}^{2+}$ составляют $2.27,-2.42$ и -2.62 В (отн. НВЭ), соответственно. В результате установлено, что УФ облучение реакционных растворов, полученных после взаимодействия кристаллогидратов $\mathrm{LnCl}_{3} \cdot 6 \mathrm{H}_{2} \mathrm{O}(\mathrm{Ln}=\mathrm{Tm}, \mathrm{Dy}$, $\mathrm{Nd})$ с ${ }^{i} \mathrm{Bu}_{2} \mathrm{AlH}$ в ТГФ, не приводит к восстановлению этих ионов $\mathrm{Ln}^{3+}$ до $\mathrm{Ln}^{2+}$. Исходя из этих результатов, значение восстановительного потенциала ${ }^{i} \mathrm{Bu}_{2} \mathrm{AlH}$ находится между -1.55 и $-2.27 \mathrm{~B}$.

\section{Выводы}

С целью разработки новых, более эффективных методов синтеза растворимых комплексов ионов двухвалентных лантанидов $\mathrm{Ln}^{2+}$, перспективных в качестве катализаторов и одноэлектронных восстановителей, в работе изучена способность ионов $\mathrm{Ln}^{3+}$ к восстановлению до ионов $\mathrm{Ln}^{2+}$, где $\mathrm{Ln}$ $=\mathrm{Sm}, \mathrm{Tm}, \mathrm{Dy}, \mathrm{Nd}$, при взаимодействии с $\mathrm{AOC}$ $\left({ }^{i} \mathrm{Bu}_{2} \mathrm{AlH},{ }^{i} \mathrm{Bu}_{3} \mathrm{Al}, \mathrm{Et}_{3} \mathrm{Al}\right)$ в ТГФ под действием микроволнового, ультрафиолетового и ультразвукового облучения. Обнаружено, что процесс восстановления самария $\mathrm{Sm}^{3+}$ до $\mathrm{Sm}^{2+}$ под действием $\mathrm{AOC}$, характеризующийся существенной длительностью 
процесса (2-24 ч), значительно ускоряется действием УФ света. Так, при УФ облучении раствора, образующегося после взаимодействия в системе $\mathrm{SmCl}_{3} \cdot 6 \mathrm{H}_{2} \mathrm{O}-{ }^{i} \mathrm{Bu}_{2} \mathrm{AlH}-\mathrm{TГ}$, восстановление $\mathrm{Sm}^{3+} \rightarrow$ $\mathrm{Sm}^{2+}$ осуществляется за рекордные 15 мин. В то же время в аналогичных условиях УФ облучения другие ионы лантанидов $\mathrm{Tm}^{3+}, \mathrm{Dy}^{3+}, \mathrm{Nd}^{3+}$ не восстанавливаются из-за их гораздо более отрицательных восстановительных потенциалов по сравнению с ионом $\mathrm{Sm}^{3+}$.

Исследование проведено в соответствии с бюджетными темами ИНК УФИЦ РАН и ИФМК УФИЦ РАН.

\section{ЛИТЕРАТУРА}

1. Gopalaiah K., Kagan H. B. Use of samarium diiodide in the field of asymmetric synthesis // New J. Chem.2008. Vol. 32. P. 607-637.

2. Maity S., Flowers R. A. Mechanistic study and development of catalytic reactions ofSm(II) // J. Am. Chem. Soc.2019. Vol. 141. P. 3207-3216.

3. MolanderG. A., McKie J. A. A facile synthesis of bicyclo[m.n.1]alkan-1-ols. Evidence for organosamarium intermediates in the samarium(II) iodide promoted intramolecular Barbiertype reaction // J. Org. Chem.1991. Vol. 56. P. 4112-4120.

4. Mellah M., Bazzi S., Le Duc G., Schulz E., Gosmini, C. $\mathrm{CO}_{2}$ activation by electrogenerated divalent samarium for aryl halides carboxylation // Org. Biomol. Chem. 2019. Vol. 17. P. 8546-8550

5. Meijerink A., Dirksen G. J. Spectroscopy of divalent samarium in $\mathrm{LiBaF}_{3} / /$ Lumin. 1995. Vol. 63. P. 189-201.

6. Ramirez D., Gallagher A., Baumbach R., Siegrist T. Synthesis and characterization of the divalent samarium Zintl-phases $\mathrm{SmMg}_{2} \mathrm{Bi}_{2}$ and $\mathrm{SmMg}_{2} \mathrm{Sb}_{2} / /$ J. Solid State Chem. 2015. Vol. 231. P. 217-222.

7. Radzhabov E. A. Spectroscopy of divalent samarium in alkaline-earth fluorides // Opt. Mater.2018. Vol. 85. P. 127-132.

8. Drozdowski C. H., Gharavi-Naeini J., Stump N. A. Luminescence study of the samarium(II) sites in samarium: barium octaborate as differentiated by excitation wavelength and temperature // Appl. Spectrosc. 2017. Vol. 71. P. 1684-1688.

9. Okaue Y., Isobe T. Characterizations of divalent lanthanoid iodides in tetrahydrofuran by UV-Vis, fluorescence and ESR spectroscopy // Inorg. Chim. Acta. 1988. Vol. 144. P. 143-146.

10. Starynowicz P. Two complexes of Sm(II) with crown ethers electrochemical synthesis, structure and spectroscopy // Dalton Trans. 2004. P. 825-832.

11. Булгаков Р. Г., Елисеева С. М., Галимов Д. И. ВосстановлениеLn" ${ }^{\text {III }}$ доLn ${ }^{\text {"вреакцииLnCl }} 3 \cdot 6 \mathrm{H}_{2} \mathrm{O}(\mathrm{Ln}=\mathrm{Eu}$, $\begin{array}{lllll}\mathrm{Yb}, \quad \mathrm{Sm}) \quad \mathrm{c} & \mathrm{Bu}_{2}{ }_{2} \mathrm{AlH} & \mathrm{B} T \Gamma \Phi & \mathrm{c}\end{array}$ образованиемрастворимыхлюминесцирующихкомплексов $\mathrm{LnCl}_{2} \cdot \mathrm{xTHF} / /$ Изв. АН. Сер. Химия. 2013. №11. Р. 23452348 .

12. Butement F. D. S. Absorptionandfluorescencespectraofbivalentsamarium, europiumandytterbium // J. Chem. Soc. Faraday Trans. 1948. №309. P. 617-626.

13. Meyer G. The reduction of rare-earth metal halides with unlike metals-wöhler'smetallothermic reduction // Z. Anorg. Allg. Chem. 2007. Vol. 633. P. 2537-2552.

14. Бочкарев М. Н., Фагин А. А. Способ получения дииодидов лантаноидов (варианты) / Патент РФ №2245302 от 24.12.2003.

15. Bulgakov R. G., Eliseeva S. M., Galimov D. I. The first example of generation and emission of divalent $\mathrm{Sm}^{2+*}$ ion in a liquid-phase chemiluminescence in the system $\mathrm{SmCl}_{3} \cdot 6 \mathrm{H}_{2} \mathrm{O}-$
THF-Bu ${ }_{2}^{\mathrm{i}} \mathrm{AlH}-\mathrm{O}_{2} / /$ J. Photochem. Photobiol. A: Chem. 2015. Vol. 300. P. 1-5.

16. Yuan Y., Zhao W., Liu Z., Ling C., Zhu C., Liu F., Li A., Low-Fe(III) driven UV/Air process for enhanced recovery of heavy metals from EDTA complexed system // Water Res. 2020. Vol. 171. P. 115375

17. Van den Bogaert B., Havaux D., Binnemans K., Van Gerven T. Photochemical recycling of europium from Eu/Y mixtures in red lamp phosphor waste streams // Green Chem.2015. Vol. 17. P. 2180-2187.

18. Xie H., Zhang L., Li H., Li S., Chen K., Zhang B., Zhou M. Ultrasonic-enhanced replacement of lead in lead hydrometallurgy process from lead leaching solution // R. Soc. Open Sci.2019. Vol. 6. P. 190042.

19. Kim B.-J., Cho K., Lee S.-G., Park C.-Y., Choi N.-C., Lee S. Effective gold recovery from near-surface oxide zone using reductive microwave roasting and magnetic separation // Metals. 2018. Vol. 8. P. 957.

20. Bulgakov R. G., Eliseeva S. M., Galimov D. I. Peculiarities of bright blue liquid-phase chemiluminescence of the $\mathrm{Eu}^{2+*}$ ion generated at interactions in the systems $\mathrm{EuX}_{3} \cdot 6 \mathrm{H}_{2} \mathrm{O}-\mathrm{THF}-\mathrm{R}_{3}$ ${ }_{\mathrm{n}} \mathrm{AlH}_{\mathrm{n}}-\mathrm{O}_{2}\left(\mathrm{X}=\mathrm{Cl}, \mathrm{NO}_{3} ; \mathrm{R}=\mathrm{Bu}^{\mathrm{i}}, \mathrm{Et}\right.$ and $\left.\mathrm{Me} ; \mathrm{n}=0,1\right) / / \mathrm{J}$. Lumin. 2016. Vol. 172. P. 71-82.

21. Girard P., Namy J. L., Kagan H. B. Divalent lanthanide derivatives in organic synthesis. 1. Mild preparation of samarium iodide and ytterbium iodide and their use as reducing or coupling agents // J. Am. Chem. Soc.1980. Vol. 102. P. 2693-2698.

22. Полуэктов Н. С., Кононенко Л. И., Ефрюшина Н. П., Бельтюкова С. В. Спектрофотометрические и люминесцентные методы определения лантаноидов / Киев: Наукова думка, 1989. 255 с.

23. Каменская А. Н. Низшее состояние окисления лантанидов в растворах // Ж. неорган. химии. 1984. Т. 29. Вып. 2. С. 439-449.

24. Morss L. R. Thermochemical properties of yttrium, lanthanum, and the lanthanide elements and ions // Chem. Rev. 1976. Vol. 76. P. 827-841

25. Evans W. J., Champagne T. M., Giarikos D. G., Ziller J. W. Lanthanide metallocene reactivity with dialkyl aluminum chlorides: modeling reactions used to generate isoprene polymerization catalysts // J. Organomet. Chem. 2005. Vol. 24. P. 570-579.

26. Бочкарев М. Н., Калинина Г. С., Захаров Л. Н., Хоршев С. Я. Органические производные редкоземельных элементов. М.: Наука, 1989. 232 с.

27. Рафиков С. Р., Монаков Ю. Б., Биешева Я. Х., Валитова И. Ф., Муринов Ю. И., Толстиков Г. А., Никитин Ю. Е. Полимеризация изопрена на соединениях подгруппы лантана // Докл. АН СССР. 1976. Т. 229. №5. С. 1174-1176.

28. Shen Z., Ouyang J., Yu F., Hu Z., Yu F., Qian B. The characteristics of lanthanide coordination catalysts and the cispolydienes prepared therewith // J. Polym. Sci., Part A: Polym. Chem. 1980. Vol. 18. P. 3345-3357.

29. Evans W. J., Gummersheimer T. S., Ziller J. W. Coordination chemistry of samarium diiodide with ethers including the crystal structure of tetrahydrofuran-solvated samarium diiodide, $\mathrm{SmI}_{2}(\mathrm{THF})_{5} / /$ J. Am. Chem. Soc. 1995. Vol. 117. P. 8999-9002.

30. Watson P. L., Tulip T. H., Williams I. Defluorination of perfluoroolefins by divalent lanthanoid reagents: activating $\mathrm{C}$ F bonds // Organometallics. 1990. Vol. 9. P. 1999-2009.

31. Hechimann G., Niemever M. Synthesis and first structural characterization of lanthanide(II) aryls: observation of a schlenk equilibrium in europium(II) and ytterbium(II) chemistry // J. Am. Chem. Soc. 2000. Vol. 122. P. 4227-4230.

32. Brum J. L., Deshmukh S., Koplitz B. Substantial H-atom production from the $193 \mathrm{~nm}$ photolysis of triethylaluminum // Chem. Phys. Lett.1990. Vol. 165. P. 413-416.

33. Herley P. J., Spencer D. H. Photolytic decomposition of lithium aluminum hydride powder // J. Phys. Chem. A. 1979. Vol. 83. P. 1701-1707. 
DOI: $10.33184 /$ bulletin-bsu-2020.3.9

\title{
PHOTOCHEMICAL ACTIVATION OF THE REDUCTION PROCESS $\mathrm{OF} \mathrm{Sm}^{3+}$ TO $\mathrm{Sm}^{2+}$ IN THE $\mathrm{SmCl}_{3} \cdot 6 \mathrm{H}_{2} \mathrm{O}-{ }_{-}^{\mathrm{I}} \mathrm{Bu}_{2} \mathrm{AlH}-\mathrm{THF}$ SYSTEM
}

\author{
() D. I. Galimov ${ }^{1}$, S. M. Yakupova ${ }^{1}$, R. G. Bulgakov ${ }^{2}$ \\ ${ }^{1}$ Institute of Petrochemistry and Catalysis, Ufa Federal Research Center of RAS \\ 141 Oktyabrya Avenue, 450075 Ufa, Republic of Bashkortostan, Russia. \\ ${ }^{2}$ Institute of Molecular and Crystal Physics, Ufa Federal Research Center of RAS \\ 71 Oktyabrya Avenue, 450054 Ufa, Republic of Bashkortostan, Russia. \\ Phone: +7 (347) 2842750 . \\ *Email: galimovdi@mail.ru
}

The ability of the trivalent lanthanide ions $\operatorname{Ln}^{3+}(\mathrm{Ln}=\mathrm{Sm}, \mathrm{Tm}, \mathrm{Dy}, \mathrm{Nd})$ to be reduced to a divalent state at the interaction with organoaluminum compounds $\left({ }^{\mathrm{i}} \mathrm{Bu}_{2} \mathrm{AlH},{ }^{\mathrm{i}} \mathrm{Bu}_{3} \mathrm{Al}\right.$, $\mathrm{Et}_{3} \mathrm{Al}$ ) in an aprotic solvent tetrahydrofuran under the action of microwave, ultraviolet and ultrasonic irradiation were studied for the first time in order to develop the new effective synthesis methods of soluble complexes of the divalent lanthanides ions $\mathrm{Ln}^{2+}$, promising as catalysts and one-electron reductants. Using the chemical, elemental, and spectral methods of analyzes, the composition of the obtained complex of divalent samarium $\mathrm{SmCl}_{2}(\mathrm{THF})_{2}$ was established. The spectra of photoluminescence, PL excitation, and absorption of $\mathrm{Sm}^{2+}$ ions in the reaction solution and in individual form were measured. It was found that the reduction process of samarium $\mathrm{Sm}^{3+}$ to $\mathrm{Sm}^{2+}$ under the action of ${ }^{\mathrm{i}} \mathrm{Bu}_{2} \mathrm{AlH}$, characterized by a long duration of process from 2 to 24 hours, is significantly accelerated by exposure to UV light. Under UV irradiation, the solution formed after interaction in the $\mathrm{SmCl}_{3} \cdot 6 \mathrm{H}_{2} \mathrm{O}$ ${ }^{\mathrm{i}} \mathrm{Bu}_{2} \mathrm{AlH}-\mathrm{THF}$ system. The reduction of $\mathrm{Sm}^{3+} \rightarrow \mathrm{Sm}^{2+}$ was carried out in a record 15 minutes. The reduction process was monitored by the absorption and photoluminescence spectra of $\mathrm{Sm}^{2+}$ in the reaction solution. It was found that the reduction of other lanthanide ions $\mathrm{Ln}^{3+}(\mathrm{Ln}=\mathrm{Tm}, \mathrm{Dy}, \mathrm{Nd})$ under the action of ${ }^{\mathrm{i}} \mathrm{Bu}_{2} \mathrm{AlH}$ and irradiation does not occur, which is associated with large negative reduction potentials $\mathrm{E}^{0}\left(\mathrm{Ln}^{3+} / \mathrm{Ln}^{2+}\right) \leq-2.27 \mathrm{~V}$ for this lanthanide ions.

Keywords: divalent samarium ion, photochemical synthesis, $\mathrm{Sm}^{3+}$ reduction, photoluminescence, UV irradiation.

Published in Russian. Do not hesitate to contact us at bulletin_bsu@mail.ru if you need translation of the article.

\section{REFERENCES}

1. Gopalaiah K., Kagan H. B. New J. Chem.2008. Vol. 32. Pp. 607-637.

2. Maity S., Flowers R. A. J. Am. Chem. Soc.2019. Vol. 141. Pp. 3207-3216.

3. MolanderG. A., McKie J. A. J. Org. Chem.1991. Vol. 56. Pp. 4112-4120.

4. Mellah M., Bazzi S., Le Duc G., Schulz E., Gosmini, C. Pp. 2activation by electrogenerated divalent samarium for aryl halides carboxylation. Org. Biomol. Chem. 2019. Vol. 17. Pp. 8546-8550.

5. Meijerink A., Dirksen G. J. Lumin. 1995. Vol. 63. Pp. 189-201.

6. Ramirez D., Gallagher A., Baumbach R., Siegrist T. J. Solid State Chem. 2015. Vol. 231. Pp. $217-222$.

7. Radzhabov E. A. Opt. Mater.2018. Vol. 85. Pp. 127-132.

8. Drozdowski C. H. Appl. Spectrosc. 2017. Vol. 71. Pp. 1684-1688.

9. Okaue Y., Isobe T. Inorg. Chim. Acta. 1988. Vol. 144. Pp. 143-146.

10. Starynowicz P. Dalton Trans. 2004. Pp. 825-832.

11. Bulgakov R. G., Eliseeva S. M., Galimov D. I. Izv. AN. Ser. Khimiya. 2013. No. 11. Pp. 2345-2348.

12. Butement F. D. S. J. Chem. Soc. Faraday Trans. 1948. No. 309. Pp. 617-626.

13. Meyer G. Z. Anorg. Allg. Chem. 2007. Vol. 633. Pp. 2537-2552.

14. Bochkarev M. N., Fagin A. A. Sposob polucheniya diiodidov lantanoidov (varianty) / Patent RF No. 2245302 ot 24.12 .2003

15. Bulgakov R. G., Eliseeva S. M., Galimov D. I. J. Photochem. Photobiol. A: Chem. 2015. Vol. 300. Pp. 1-5.

16. Yuan Y., Zhao W., Liu Z., Ling C., Zhu C., Liu F., Li A. Water Res. 2020. Vol. 171. Pp. 115375.

17. Van den Bogaert B., Havaux D., Binnemans K., Van Gerven T. Green Chem.2015. Vol. 17. Pp. $2180-2187$.

18. Xie H., Zhang L., Li H., Li S., Chen K., Zhang B., Zhou M. R. Soc. Open Sci.2019. Vol. 6. Pp. 190042.

19. Kim B.-J., Cho K., Lee S.-G., Park C.-Y., Choi N.-C., Lee S. Metals. 2018. Vol. 8. Pp. 957. 
20. Bulgakov R. G., Eliseeva S. M., Galimov D. I. J. Lumin. 2016. Vol. 172. Pp. 71-82.

21. Girard P., Namy J. L., Kagan H. B. J. Am. Chem. Soc.1980. Vol. 102. Pp. 2693-2698.

22. Poluektov N. S., Kononenko L. I., Efryushina N. P., Bel'tyukova S. V. Spektrofotometricheskie i lyuminestsentnye metody opredeleniya lantanoidov [Spectrophotometric and luminescent methods for the determination of lanthanides] / Kiev: Naukova dumka, 1989.

23. Kamenskaya A. N. Zh. neorgan. khimii. 1984. Vol. 29. No. 2. Pp. 439-449.

24. Morss L. R. Chem. Rev. 1976. Vol. 76. Pp. 827-841.

25. Evans W. J., Champagne T. M., Giarikos D. G., Ziller J. W. J. Organomet. Chem. 2005. Vol. 24. Pp. 570-579.

26. Bochkarev M. N., Kalinina G. S., Zakharov L. N., Khorshev S. Ya. Organicheskie proizvodnye redkozemel'nykh elementov [Organic derivatives of rare earth elements]. Moscow: Nauka, 1989.

27. Rafikov S. R., Monakov Yu. B., Biesheva Ya. Kh., Valitova I. F., Murinov Yu. I., Tolstikov G. A., Nikitin Yu. E. Dokl. AN SSSR. 1976. Vol. 229. No. 5. Pp. 1174-1176.

28. Shen Z., Ouyang J., Yu F., Hu Z., Yu F., Qian B. J. Polym. Sci., Part A: Polym. Chem. 1980. Vol. 18. Pp. 3345-3357.

29. Evans W. J., Gummersheimer T. S., Ziller J. W. J. Am. Chem. Soc. 1995. Vol. 117. Pp. 8999-9002.

30. Watson P. L., Tulip T. H., Williams I. Organometallics. 1990. Vol. 9. Pp. 1999-2009.

31. Hechimann G., Niemever M. J. Am. Chem. Soc. 2000. Vol. 122. Pp. 4227-4230.

32. Brum J. L., Deshmukh S., Koplitz B. Chem. Phys. Lett.1990. Vol. 165. Pp. 413-416.

33. Herley P. J., Spencer D. H. J. Phys. Chem. A. 1979. Vol. 83. Pp. 1701-1707. 